\title{
Energy-Aware Adaptation of Educational Multimedia in Mobile Learning
}

\author{
Syed Asim Jalal, Nicholas Gibbins, David Millard, Bashir Al-Hashimi, Naif Radi Aljohani \\ School of Electronics and Computer Science \\ University of Southampton \\ SO171BJ, Southampton, United Kingdom \\ \{smaj08r,nmg,dem,bmah,nra1d10\}@ecs.soton.ac.uk
}

\begin{abstract}
As a result of tremendous enhancements in the capabilities of mobile devices and availability of higher data rate mobile internet, the use of online multimedia learning resources on mobile devices is increasingly becoming popular. Limited Battery Power of mobile devices, however, is still one big challenge in Mobile Learning. High Quality multimedia learning resources are power hungry and if used on mobile devices drain battery power rapidly limiting learning opportunities on the move. Lack of significant improvements in battery capacities has resulted in significant interest in battery power saving techniques. Existing power-saving streaming multimedia adaptation techniques tend to extend battery life by reducing quality of multimedia making them susceptible to information loss. This loss may affect the learning content efficacy and jeopardizes the learning process. To the best of our knowledge, no previous work has considered the learning content efficacy in multimedia streaming adaptation mechanism. In this paper, we present MoBELearn system, which is a prototype implementation of our proposed Content Aware Power Saving Educational Multimedia Adaptation (CAPS-EMA) approach. We demonstrate battery efficiency in educational multimedia streaming while keeping the adapted resource suitable for learning. We also describe our semantic metamodel for educational multimedia resource that support our energy efficient adaptation technique.
\end{abstract}

\section{Categories and Subject Descriptors}

K.3.1 Computer Uses in Education, Distance learning

\section{General Terms}

Performance, Design, Human Factors

\section{Keywords}

Mobile Learning, Energy Efficiency, Multimedia Learning, Battery Saving

\section{INTRODUCTION}

In recent years Mobile Learning has become very popular. Powerful mobile devices with much enhanced capabilities are now becoming more affordable. Furthermore, tremendous improvements in wireless technologies have made it possible to connect these mobile devices with internet at higher data rates, enabling the use of high quality multimedia content possible. As a result mobile users have now access to rich educational multimedia content anytime and anywhere [1]. Further opportunities of learning using mobile devices have been created by the free availability of huge number educational videos by many reputed institutions and individuals. Mobile Learners have now control over what to learn, when and where to learn making learning using mobile devices increasingly popular [2, 3]. There are, however, some challenges that still need to be addressed.

There is big diversity in terms of mobile devices, network connectivity as well as mobile learners' needs. This makes the traditional one-size-fit-all approach unsuitable for the mobile learning resources. Adaptation and personalization of learning resources has been used to enhance and make the learning experience more efficient. It is very important to facilitate mobile learner in taking better advantage of the limited time and resource constrained device while on the move. Mobile devices have resource constraints in features like screen size, network characteristics, memory size and battery-power. There has been much research in adapting learning resources to the learner's needs and usage context [4] [5] [6]. The use of multimedia content specially poses many challenges in the delivery of mobile Learning context. Multimedia adaptation and personalization techniques have also been developed for Mobile Learning systems [3].

Traditionally, Content Adaptation and Universal Multimedia Access (UMA) [7] techniques have been used to adapt multimedia content based on the resource constraints of mobile devices. Battery technology has not seen as much improvement as other features of mobile devices. Mobile devices have limited battery power which restricts learner from learning for longer duration. Educational multimedia content on the web when accessed over the mobile network quickly drains battery power as result of larger data transfer. Wireless Interface of Mobile Devices consumes major portion of battery in multimedia streaming. Streaming multimedia can consume up to two times more battery power than playing the same multimedia file locally $[8,9]$. Energy efficiency in this applications area has therefore been addressed by many researchers. The techniques developed so far, however, are not suitable for adapting educational multimedia as they do not consider learning factor in the 
adaptation process. It is only recently that Power Saving techniques have been recommended to be integrated in Mobile Learning environments to enable learning experience [10, 11].

Energy efficient multimedia adaptation techniques tend to degrade multimedia quality in order to extend the battery life [12] and are, therefore, based on trade-off between user experience and battery life. Multimedia quality is lowered by reducing the encoding parameters like Frame per second, bitrates, colors and resolution. Reducing quality of educational multimedia resources beyond a certain quality can leave the resource unsuitable for learning due to the loss of crucial quality sensitive information. There is need for energy efficient educational multimedia adaptation techniques that keep multimedia suitable for learning. In our earlier work [11], we proposed a Content-aware Power Save Educational Multimedia Adaptation (CAPS-EMA) approach that achieves battery efficiency without risking the learning process.

We present a prototype implementation of our proposed novel CAPS-EMA approach. We show how to ensure maximum battery efficiency in a learning multimedia streaming application on mobile devices while keeping the multimedia useful for learning.

This paper is organized as follow. In Section II we discuss power efficiency in multimedia. In Section III we describe Adaptive Mobile Learning and Battery Efficiency. In Section IV Energy Efficient Educational Multimedia Adaptation has been discussed and in Section $\mathrm{V}$ we describe the implementation of MoBELearn System. Finally in section VI we briefly describe our Semantic Multimedia Learning Resource Model.

\section{POWER EFFICIENCY IN MULTIMEDIA STREAMING}

On one hand advancements in battery capacity has lagged significantly compared to other capabilities of mobile devices [13] and on the other hand the power requirements of mobile devices are continuously increasing as mobile devices now come with increased processing capabilities, come with bigger screens and can communicate at higher data rates. Multimedia Streaming is one very power hungry application area. Considerable research has been done to improve battery efficiency while using online multimedia content. Multimedia adaptation techniques achieve power-saving by decreasing the media parameters like Frame per second, resolution, color, bitrates [14], [9] and changing even the modality of content. The general principle behind these approaches is to reduce the data size of multimedia which reduces the data transfer through wireless interface and results in usage of lesser battery power. A useful survey of Energy efficient techniques for multimedia applications can be found in [12].

McMullin et al. introduces a Power Save-based Adaptive Multimedia Delivery (PS-AMy) mechanism [15]. PS-AMy adapts multimedia streams in order to enable the streaming last longer. This system selects stream quality based on the remaining battery life and packet loss. Other non-application layer power saving approaches include multimedia network traffic shaping which uses Network Interface Card's Sleep and Awake modes[16] [17] [18] and screen's brightness [19] during playback.

The fact that these generic multimedia streaming techniques do not consider learning efficacy of multimedia creates certain problems when used for educational resources. These problems have been discussed in detail in [11].

\section{BATTERY EFFICIENCY AND ADAPTIVE MOBILE LEARNING}

Transforming learning resources in suitable versions in order to be efficiently delivered to meet the diverse needs of learners and mobile devices is a research area that is increasingly attracting a great deal of attention. Adaptive Mobile Learning uses content adaptation and personalization techniques to provide multiple personalized versions of the same learning content [20] [4]. These techniques uses Learner's preferences, knowledge level, learning styles, the learning context (e.g., location, time, current activity, etc.), and hardware resources and constraints including screen size, resolution, processing capacity, memory, software, and network connectivity to personalize learning resources.

Qing Tan in [5] Proposes a 5R adaptation framework, the aim of which is to provide a learning resource "at the right time, in the right location, through the right device, providing the right contents to the right learner". [21] discusses some issues arising due to the diversity that exists in learning on mobile devices. Over the years, many techniques have been proposed to provide adapted versions of learning resources based on user preferences, devices characteristics and user needs. Some of these techniques can be found in [6, 22-25].

Battery power constraint is a great challenge for mobile learning. Despite high dependency on battery power little has been done to improve battery efficiency in Mobile Learning Application. Moldovan et al in [26] emphasized that power saving techniques should be integrated in multimedia learning systems. Author have shown how multimedia encoding parameters effects battery life, however, focus is on user experience while recommending encoding parameters. The same authors in [27] suggest that significant battery power can be saved using their proposed content adaptation solution in Eco-Learn m-learning system. Subjective tests conducted on participants have shown that battery power saving through multimedia quality reduction does not significantly affect learning experience and the learning outcome. Their technique recommends learning multimedia in single uniform quality. Moreover, the learning contents used in experiments were from 20-30 seconds duration. We argue that the recommended quality may be suitable for the selected short segment but there is no evidence that the same quality will remain suitable for entire educational multimedia, which may span up to an hour in most cases now a days. 
Jalal et al in [28] have also discusses battery efficiency in mobile learning context. This work, however, is targeted at enabling the discovery of battery efficient version of an adaptive learning resource among many available adaptive contents online.

Moldovan et al in [10] gives a detailed survey of methods and techniques that can be used to achieve energy efficiency in mobile learning.

We can see that mobile learning applications for multimedia adaptation rely on existing generic adaptation techniques. These techniques do not consider the learning aspect of multimedia. We, therefore, emphasize on the need of multimedia adaptation techniques specifically developed for educational multimedia that consider the effect of adaptation on learning outcomes. To the best of our knowledge, our approach mentioned in [11] is the first one to consider the effect of multimedia adaptation on learning experience as opposed to user experience.

\section{ENERGY EFFICIENT EDUCATIONAL MULTIMEDIA ADAPTATION}

Based on the identified shorting comings in the existing techniques, we presented a Content-Aware Power-Saving Educational Multimedia Adaptation (CAPS-EMA) mechanism [11]. This mechanism promises to provide optimum battery efficiency while keeping the adapted content suitable for learning. In this approach the entire multimedia content is fragmented with multiple quality versions. We consider the learning aspect of content in the adaptation by determining minimum acceptable quality for each fragment that will retain the fragment suitable for learning purposes. Then these minimum useful qualities are placed as constraints for respective fragment in metadata. If a fragment of multimedia has quality sensitive visual contents then it will be assigned a higher quality presentation constraint. On the other hand if another fragment of the video does not contain any important visual information at all and all the information is oral then the presentation constraint for such fragment can be audio quality. The idea is to deliver each portion of multimedia at minimum possible energy cost.

This adaptation mechanism is based on the fact that most learning videos present visual information of different details at different temporal point in the multimedia resource. Visual content varies in size, colors, detail and motion. Our proposed approach considers the effect of quality reduction on the visual information that is contained in each temporal fragment, by imposing quality constraints. This makes the minimum video quality requirement variable at different temporal points of multimedia content. A fragment in our solution's context is a portion of multimedia segment in the temporal dimension that has similar minimum presentation quality requirement to effectively achieve the learning aims. The output of our CAPS-EMA approach is a fragmented, multi-quality and multimodal format as show in Figure 1, which compares the output with existing techniques.

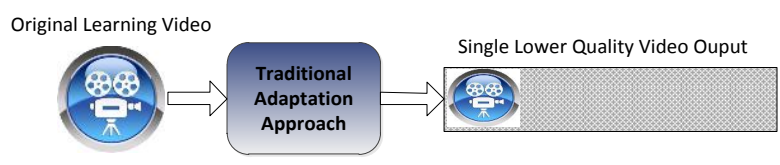

(a)- Existing Adaptation Approaches

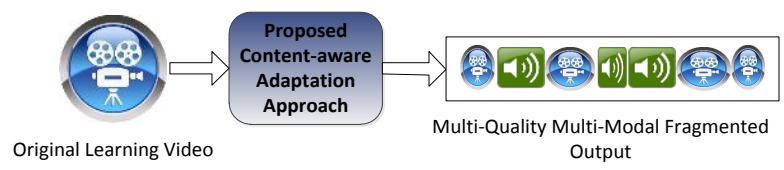

(b)- Proposed Content Aware Adaptation Approach

Figure 1: Comparison of Existing and CAPS-EMA [11] Approaches

\section{MOBELEARN SYSTEM}

In this section, we present a prototype implementation MoBELearn (MObile Battery Efficient LEARNing) based on the CAPS-EMA approach. Figure 2 describes architecture of the prototype. MoBELearn consists of a Media Store that contains learning multimedia consisting of original high quality videos and their fragments in different quality versions. A subset of the fragmented versions is selected during adaptation and delivered to the user device. In our prototype we have used MP4 and MP3 media. Original videos are manually fragmented and encoded into different versions. It can be a good future research direction to investigate how we can automatically fragment multimedia resource based on similar minimum acceptable qualities and create suitable verisons.

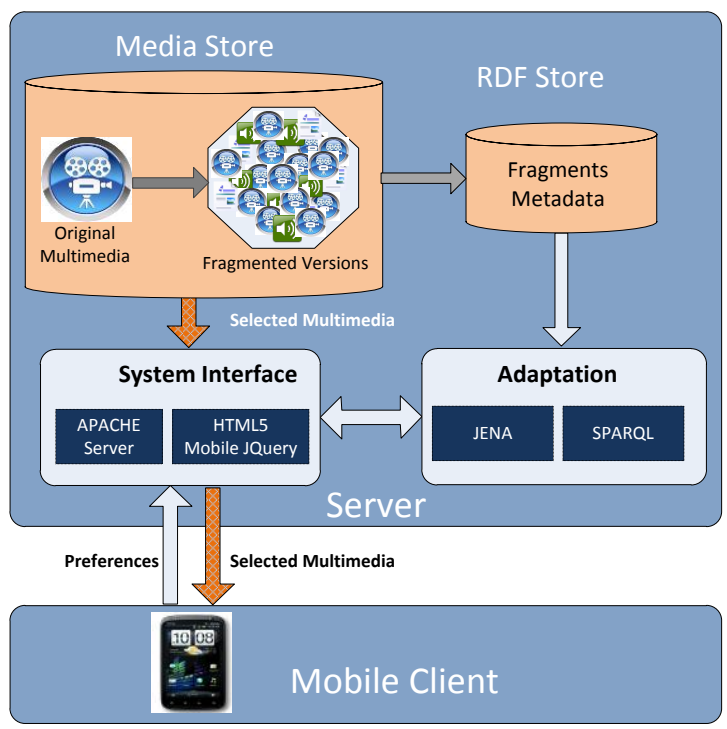

Figure 2: MoBELearn Architecture

The System Interface component of the system is for user interaction. Learners can specify energy saving preferences and retrieve learning content. The interface component is developed using HTML5, Mobile JQuery and is deployed on Apache Webserver. The Adaptation Process consists of generating 
SPARQL Query that will be used to retrieve the URLs of appropriate media fragments. Multimedia Metadata component makes major component of our approach. We have implemented the metadata model as Resource Description Framework (RDF) model. The meta-model is explained in section 6 .

\begin{tabular}{|c|c|}
\hline Q. $\mathrm{H}$.nI $7: 02 \mathrm{PM}$ & 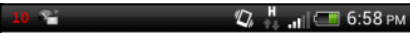 \\
\hline mvl.ecs.soton.ac.uk: 8080 & mvl.ecs. soton.ac.uk: 8080 \\
\hline $\begin{array}{l}\text { Mobile Battery Efficient } \\
\text { Learning (MOBELearn) }\end{array}$ & $\begin{array}{l}\text { Mobile Battery Efficient } \\
\text { Learning (MOBELearn) }\end{array}$ \\
\hline Test Video : Structure of Atom & Test Video :Structure of Atom \\
\hline Select Battery Power Saving Level & Select Battery Power Saving Level \\
\hline View Learning Content & View Learning Content \\
\hline & $\begin{array}{l}\text { This option will save Maximum approx. } 76 \% \\
\text { battery power in data transfer }\end{array}$ \\
\hline $\begin{array}{l}\text { You will receive entire video as a Single High } \\
\text { Quality Video. }\end{array}$ & $\begin{array}{l}\text { Learning Video Content will be displayed in } \\
\text { fragments. Content will be delievered in Lowest } \\
\text { Acceptable Quality for delivering video content } \\
\text { except where fragments require high, medium } \\
\text { or low quality video for conveying learning } \\
\text { information correctly. The minimum } \\
\text { acceptable quality will vary from fragment to } \\
\text { fragment and it can be Audio where possible }\end{array}$ \\
\hline
\end{tabular}

Figure 3: MoBELearn Interface

Figure 3, shows the user interface of the MoBELearn system. A Slide-Bar is used to allow user to specify her energy efficiency preferences. Each option in the slide-bar provides a brief feedback about the corresponding adapted Learning Resource. For example, user preference in the left screenshot will result in no battery saving, in which case, user device will be provided the original high quality video as one continuous fragment. In the right screenshot, user preference for maximum battery efficiency is selected. This will result in each fragment being delivered in its minimum acceptable quality for learning achieving maximum possible battery energy efficiency. It shows approximately $76 \%$ energy saving can be achieved during wireless data transfer. This efficiency value in our prototype has been computed beforehand for these options using PowerTutor android app [29] . It is important to note that we are not interested exactly in the values of energy consumption (in Joules) as the exact values vary in different cases and is affected by several other factors including, type of wireless technology (GSM, 3G, LTE, WiFi), carriers properties and strength of wireless signals [9]. Various battery consumption models have been proposed to measure energy expenses in terms of data transfer. Battery consumption model could be used to approximately compute battery consumption and determine efficiency. These models are based on data size of the transfer.

In our test case we used an openly available sample learning video titled "Structure of Atom" from Derek Owens's YouTube channel. We fragmented, created versions and created metadata. Quality constraints were determined after observing the content.

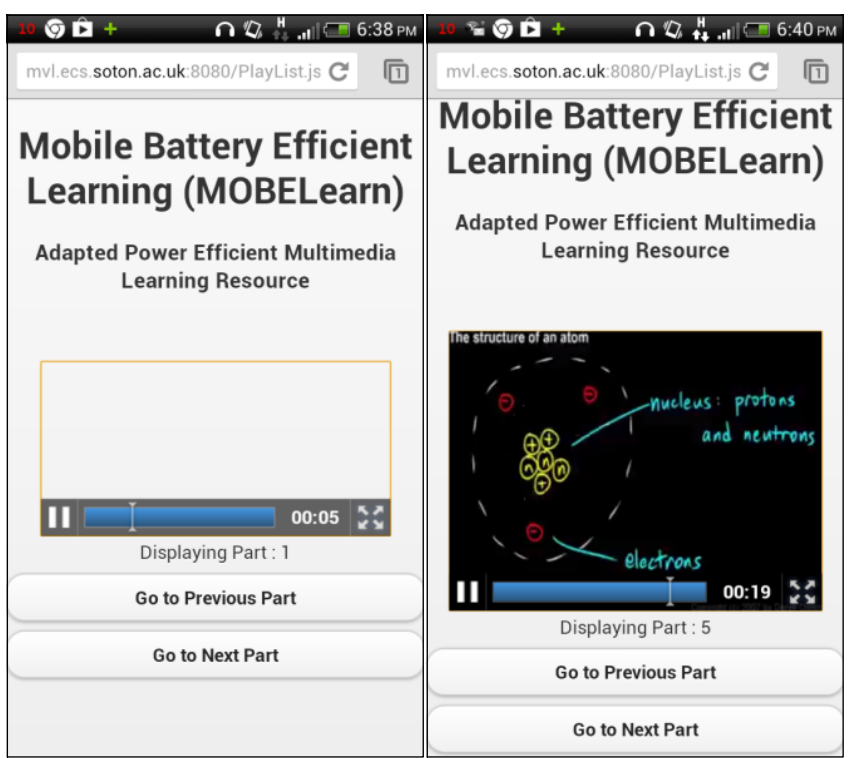

Figure 4: Screenshots of the prototype System

Figure 4 shows a sample adapted output of preferences in Figure 3. Figure 4(Left) shows fragment being delivered in Audio quality while in Figure 4 (Right) Screenshot display fragment 5 in a very low quality version.

This low Quality Version been shown along the original version in figure 7 to see the difference. We can see that the difference Video on the left has bitrate of $124 \mathrm{kbps}, 30$ Frames per Second and stereo audio quality while the video fragment on the right has bitrate of $39.1 \mathrm{kbps}, 5$ frames per second and Mono audio quality. We can see that energy saving would be further greater if the original video quality of bitrates higher than $124 \mathrm{kbps}$ is used which is very normal these days.

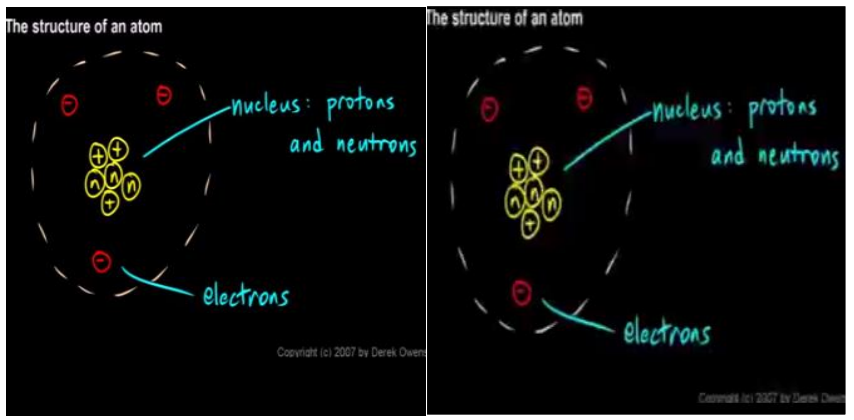

Figure 5: Comparison of Original and Adapted Content

\section{MULTIMEDIA LEARNING RESOURCE MODEL}

This adaptation approach is based on fragments' metadata. We have developed RDF based Fragmented Educational Multimedia Resource Ontology Model (FEMROM) to enable adaptation. Figure 6, shows FEMROM model. In this model, a LearningResource (Multimedia Learning Resource) is made of up of many ResourceFragments. LearningResource has common data properties like dc:identifier, dc:creater, dc:subject. To 
represent the order of fragment in the entire resource we have data property of fragment order number (FragOrderNum). Each ResourceFragments has Importance property to represent the degree of importance or usefulness of the fragment. This property can help in skipping the optional fragments to achieve further energy efficiency. As we discussed, each Fragment has a minimum acceptable quality that will keep the fragment learnable, this is achieved using hasPresentationConstraints object property that associates the QualityLevel constraints to ResourceFragments. Each ResourceFragment has many FragmentVersions using hasFragmentVersions. Each FragmentVersion has different QualityLevel and Modality. Each FragmentVersion has a separate identifier (URL), one of these version URLs will be selected for each fragment during the adaptation process. The exact quality parameters for each QualityLevel are represented by PresentationFeatures, which contains attributes like bitrate, Resolution and FPS (frame per Second).

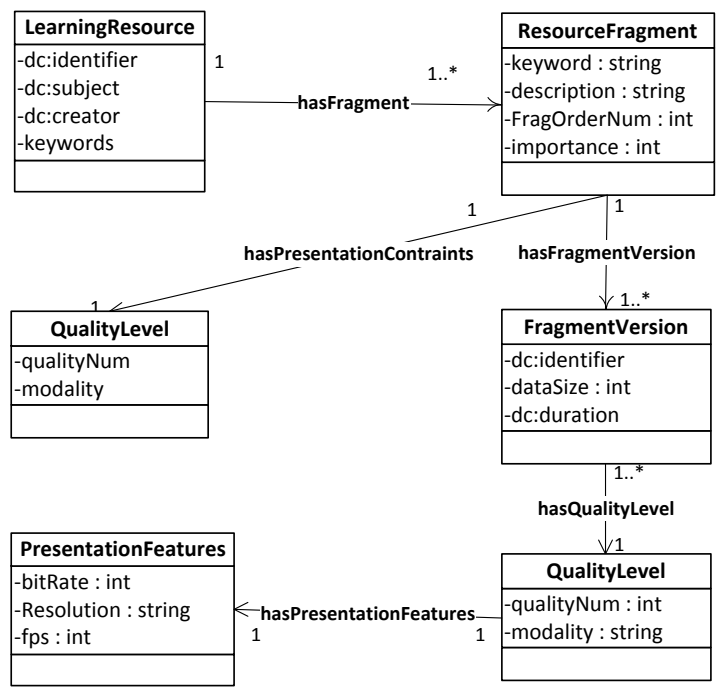

Figure 6: Educational Multimedia Metamodel

The actual Adaptation process happens at the level of selection of the fragments and their versions. This selection is done on the basis of the user preferences (User Model) about the extent of desired power efficiency and the metadata. User Preference is converted into a SPARQL query which queries RDF store to retrieve URLs of the multimedia fragments. URLs of fragments are delivered to HTML 5 video player in learner's mobile device as a playlist using MobileJQuery. On Android devices the HTML 5 Video Player has auto-play feature disabled. As a result of this, in our current implementation, learner has to click on the each media fragment to start playing, which is a user experience (UX) concern.

In Figure 7, we show a sample SPARQL query that selects Multimedia media fragments in the minimum acceptable quality to achieve maximum energy efficiency.

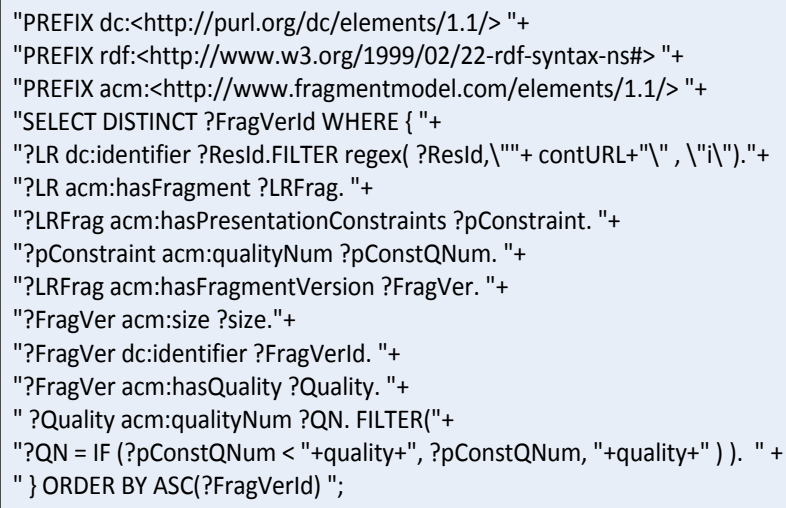

\section{Figure 7: Example SPARQL Query}

We have made provision in our meta-model to design a system that can offer further energy efficiency by selecting only the most important media fragments and exclude fragment that are not relevant to the user existing knowledge level or contain optional explanations.

\section{CONCLUSION}

We presented an implementation of our proposed novel energy efficient adaptation technique to support battery efficiency in emerging area of mobile learning. The fundamental idea is that educational effectiveness of learning multimedia should be considered in power-saving multimedia adaptation process. We presented MoBELearn system, which adapts entire multimedia resource on fragment by fragment basis considering presentation constraints on each fragment, offering many advantages over existing adaptation techniques that deliver adapted multimedia in a single uniform quality. We ensure that maximum possible battery efficiency is achieved without negatively affecting the learning process. We also presented our semantic educational multimedia learning resource model that supports our prototype system, which is implemented in RDF.

\section{REFERENCES}

1. Cheung, S.K., A study on the use of mobile devices for distance learning, in Hybrid Learning2012, Springer. p. 8998.

2. Bradley, C., et al., Design and development of multimedia learning objects for mobile phones. Mobile learning: Transforming the delivery of education and training, 2009: p. $157-182$.

3. Moldovan, A.N. and C.H. Muntean. Towards personalised and adaptive multimedia in m-learning systems. in World Conference on E-Learning in Corporate, Government, Healthcare, and Higher Education. 2011.

4. Sampson, D. and P. Zervas, Context-Aware Adaptive and Personalized Mobile Learning Systems, in Ubiquitous and Mobile Learning in the Digital Age, D.G. Sampson, et al., Editors. 2013, Springer New York. p. 3-17. 
5. Tan, Q., X. Zhang, and R.M.G. Kinshuk, The 5R Adaptation Framework for Location-Based Mobile Learning Systems, in 10th World Conference on Mobile and Contextual Learning2011: Beijing, China.

6. Zhao, X., et al., Personalized Adaptive Content System for Context-Aware Mobile Learning. Int. J. of Computer Science and Network Security, 2008. 8(8): p. 153-161.

7. Vetro, A., C. Christopoulos, and T. Ebrahimi, Universal multimedia access. IEEE Signal Processing Magazine, 2003. 20(2): p. 16.

8. Theurer, D., et al., Analysis of Individual Energy Consuming Components in a Wireless Handheld Device. 2010 ChinaIreland International Conferenceon Information and CommunicationsTechnologies (CIICT2010 E-BOOK), 2010: p. 108-112.

9. Trestian, R., et al. Energy consumption analysis of video streaming to android mobile devices. in Network Operations and Management Symposium (NOMS), 2012 IEEE. 2012. IEEE.

10. Moldovan, A., S. Weibelzahl, and C. Muntean, (accepted for publication) Energy-Aware Mobile Learning: Opportunities and Challenges. IEEE Communications Surveys and Tutorials, 2013: p. 1-32.

11. Jalal, S.A., et al. Content-aware power saving multimedia adaptation for mobile learning. in 7th International Conference on Next Generation Mobile Apps, Services and Technologies. 2013. Prague.

12. Hoque, M., M. Siekkinen, and J. Nurminen, Energy Efficient Multimedia Streaming to Mobile Devices-A Survey. IEEE Communications Surveys and Tutorials, 2012. Volume PP( Issue 99): p. 1-19.

13. Adams, J. and G.M. Muntean. Power Save Adaptation Algorithm for Multimedia Streaming to Mobile Devices. in IEEE International Conference on Portable Information Devices (PORTABLE07). 2007.

14. Moldovan, A.-N. and C.H. Muntean, Subjective Assessment of BitDetect-A Mechanism for Energy-Aware Multimedia Content Adaptation. Broadcasting, IEEE Transactions on, 2012. 58(3): p. 480-492.

15. McMullin, D., R. Trestian, and G.-M. Muntean, Power Save-based Adaptive Multimedia Delivery Mechanism, in The 9th Information Technology and Telecomunication Conference (IT\&T) 20092009

16. Korhonen, J. and Y. Wang, Power-efficient streaming for mobile terminals, in Proceedings of the international workshop on Network and operating systems support for digital audio and video2005, ACM: Stevenson, Washington, USA. p. 39-44.

17. Cornea, R., A. Nicolau, and N. Dutt, Content-aware Power Optimizations for Multimedia Streaming Over Wireless Networks, 2006, University of California.

18. Anastasi, G., et al. A power-aware multimedia streaming protocol for mobile users. in International Conference on Pervasive Services, ICPS '05. 2005.

19. Cornea, R., A. Nicolau, and N. Dutt, Using Annotations to Facilitate Power vs Quality Trade-offs in Streaming Applications, 2006: University of California.

20. Zervas, P., et al., Designing Tools for Context-Aware Mobile Educational Content Adaptation, in Ubiquitous and Mobile Learning in the Digital Age, D.G. Sampson, et al., Editors. 2013, Springer New York. p. 37-50.

21. Zhao, X. and T. Okamoto, Adaptive multimedia content delivery for context-aware u-learning. International Journal of Mobile Learning and Organisation, 2011. 5(1): p. 46-63.

22. Nguyen, V.A., V. Hanoi, and P. Van Cong. CAMLES: An adaptive mobile learning system to assist student in language learning. 2012. IEEE.

23. García, A., et al. A system for adaptation of educational contents to learners and their mobile device. 2011. IEEE.

24. Reveiu, A., I. Smeureanu, and M. Dardala. Content Adaptation in Mobile Multimedia System for M-Learning. 2008. IEEE.

25. Yang, S.J.H., I.Y.L. Chen, and R. Chen. Applying content adaptation to mobile learning. in Innovative Computing, Information and Control 2007 (ICICIC'07), Second International Conference. 2007. IEEE.

26. Moldovan, A.-N. and C.H. Muntean. Assessing Power Saving Techniques and Their Impact on E-learning Users. in 2009 IET China-Ireland International Conference on Information and Communications Technologies (CIICT 2009). 2009. National University of Ireland Maynooth.

27. Moldovan, A., A. Molnar, and C. Muntean, EcoLearn: Battery power friendly e-learning environment for mobile device users. Learning-Oriented Technologies, Devices and Networks-Innovative Case Studies, 2011: p. 273-296.

28. Asim Jalal, et al. Enabling the discovery of Adaptive Learning Resources for Mobile Learner. in 11th World Conference on Mobile and Contextual Learning: mLearn 2012. 2012. Helsinki, Finland.

29. Yang, Z., PowerTutor-A Power Monitor for Android-Based Mobile Platforms. EECS, University of Michigan, retrieved September, 2012. 2. 